\title{
Acupuncture Normalizes Dysfunction of Hypothalamic-Pituitary-Ovarian Axis
}

\author{
By \\ Bo-Ying Chen M.D. \\ Professor of Neurobiology
}

Institute of Acupuncture and Department of Neurobiology Shanghai Medical University, Shanghai 200032, P.R. China

(Received June 3, 1997; Accepted with revisions June 30,1997)

\begin{abstract}
This article summarizes the studies of the mechanism of electroacupuncture (EA) in the regulation of the abnormal function of hypothalamic pituitary-ovarian axis (HPOA) in our laboratory. Clinical observation showed that EA with the effective acupoints could cure some anovulatory patients in a highly effective rate and the experimental results suggested that EA might regulate the dysfunction of HPOA in several ways, which rneans EA could influence some gene expression of brain, thereby, normalizing secretion of some hormones, such as GnRH, LH and E2. The effects of EA might possess a relative specificity on acupoints.

KEY WORDS: Electroacupuncture, ß-Endorphin, GnRH, LH, Estradiol, Estrogen receptor, Ovariectomized rat, Hypothalamic-pituitary-ovarian axis
\end{abstract}

\section{INTRODUCTON}

Acupuncture is a treasure of Chinese traditional medicine, which is employed in the treatment of different diseases, especially in relief of all kinds of pain [1, 2] over the world. Since 1960s we have used acupuncture with appropriate electro-stimulation to cure patients with anovulation disorder (sterility), the rate of EA induction of ovulation was increased from $50 \%$ initially to $80 \%$ presently. Other authors in China also reported that acupuncture was successfully to treat patients with sterility [3] and the lying-in woman with subnormal contraction of uterus [4]. All the above research demonstrates that acupuncture may be an effective curative method of some woman's diseases. However, many questions, such as "why", "how to" and "which" about the mechanism of EA effect are unknown. To address these problems we supposed that EA might influence the production and secretion of hormones, neurotransmitters or neuro-modulators of HPOA leading to the normalization of hormone status. We also noticed certain artides reported that EA might affect the blood levels of $\mathrm{LH}, \mathrm{FSH}$, estradiol (E2) and prolactin in the female patients $[4,5,6]$ and EA may be related to long term changes in gene expression [7, 8]. These results are all significant, yet insufficient to explain the mechanism of EA in the regulation of the function of HPOA. To obtain more data, a series of experimental studies in human and animal models has been performed in our laboratory. 


\section{MATERIALS AND METHODS}

Selection and treatment of cases

Ten cases of chronically anovulatatory patients including eight cases of polycystic ovarian disease (POCA), one case of hypogonadotropic amenorrhoea and one case of oligomenorrhea were treated with EA in 13 menstruation cycles. They were all of productive age and the courses of disease were 3 to 12 years. On the 10th day of each menstruation cycle, the patients accepted the EA treatment. "Guanyuan(RN4)," "Zhongji(RN3)," "Sanyinjiao(SP6)," and bilateral "Zigong(EXCA1)" points were stimulated for $30 \mathrm{~min}$ at 8:00 AM, Q.D. for 3 days. The stimulation parameters were 7-8mA and 4-5 Hz with G6805 model generator. The electric current of EA was bearable well for every patient. The blood samples were collected from forearm of the patients one time per 15 min for detection of FSH.LH and ß-endorphin (ß-E).

Five health volunteers of a productive age with normal menstruation cycle were selected as controls, which were undergone the same treatment as above mentioned.

\section{Animals and treatments}

Wistar female rats weighting 200-250g were used. The half of animals were undergone ovariectomy and fed in the same environment with the intact rats at least for 15 days and vaginal smears were examined per day for 3 times. No exfoliative epithelium cell was found in the smears as an index for successfill ovariectomy. The ovariectomized rats and intact rats were randomly divided into two groups respectively: ovariectomized rat group (OVX), ovariectomized rat accepted EA treatment group (OVX+EA), intact rat group (INT) and intact rat accepted EA treatment group (INT+EA). The animals in OVX+EA and INT+EA received EA at the experimental acupoints of Guanyuan (RN4), Zhongji (RN3), Sanyinjiao (SP6) and bilateral Zigong (EXCA1) by EA apparatus (Model G6805-2, SMIF, Shanghai, China) with the frequency of $3 \mathrm{~Hz}$ and an intensity to produce a slight twitch of the limbs. After 3 days' treatment animals were given EA at Waiguan (SJ5) and Huatuojiaji (EXTRA21) as the control acupoints in the same way (Fig 1). By the end of last experiment, animals were sacrificed and their adrenals, brains and pituitaries were taken out for detection of nucleolar oganizer regions (AgNORs) and hormones.

Pushpull perfusion in hypothalamic preoptic area (POA) and elution of pituitary and LH and Bendorphin (ß-EP)

The technique of brain pushpull perfusion was processed as previously described by our laboratory [1]. The perfusate from hypothalamic POA was kept at $-70^{\circ} \mathrm{C}$ for GnRX and B-EP RIA.

The pituitaries were retrieved and put into $4^{\circ} \mathrm{C}$ cooled saline. Afterward, each pituitary was homogenized with $500 \mu \mathrm{l}$ of $70 \%$ acetone aqueous solution at $4^{\circ} \mathrm{C}$. The homogenate was centrifugalized $\left(2,000 \mathrm{xg}\right.$ for $15 \mathrm{~min}$ at $\left.4^{\circ} \mathrm{C}\right)$ and the supernatant was freeze-dried for $\mathrm{LH}$ and $\mathrm{B}$-EP RIA.

\section{$\underline{\text { Radioimmunoassay (RIA) of hormones }}$}

GnRH IRA: GnRH content in the perfusate from rat hypothalamus was determined by RIA method developed by Nett in 1973 [9]. GnRH was iodinated by the modified chlomine-T technique[10]. Na125 I was manufactured by Radiochemical Center, Amersham.

B-EP RIA: The sensitive radioimmunoassay was a routine in our laboratory [1]. The standards of human and rat B-EP was synthesized by Peninsula Laboratories, Inc. and the rabbit antiserum of both $ß$-EP was developed in our laboratory. The cross-reaction from human B-EP and camel ßEP was detected about $20 \%$. The sensitivity of this method was 10pg/tube.

$\underline{\mathrm{LH}}, \mathrm{E} 2$ and corticosterone RIA: LH, E2 and corticosterone RIA kits were bought from Shanghai Institute of Biologic Products, the Ministry of Health, P.R. China. All procedures of RIA were 
performed as described in the kit manuals.

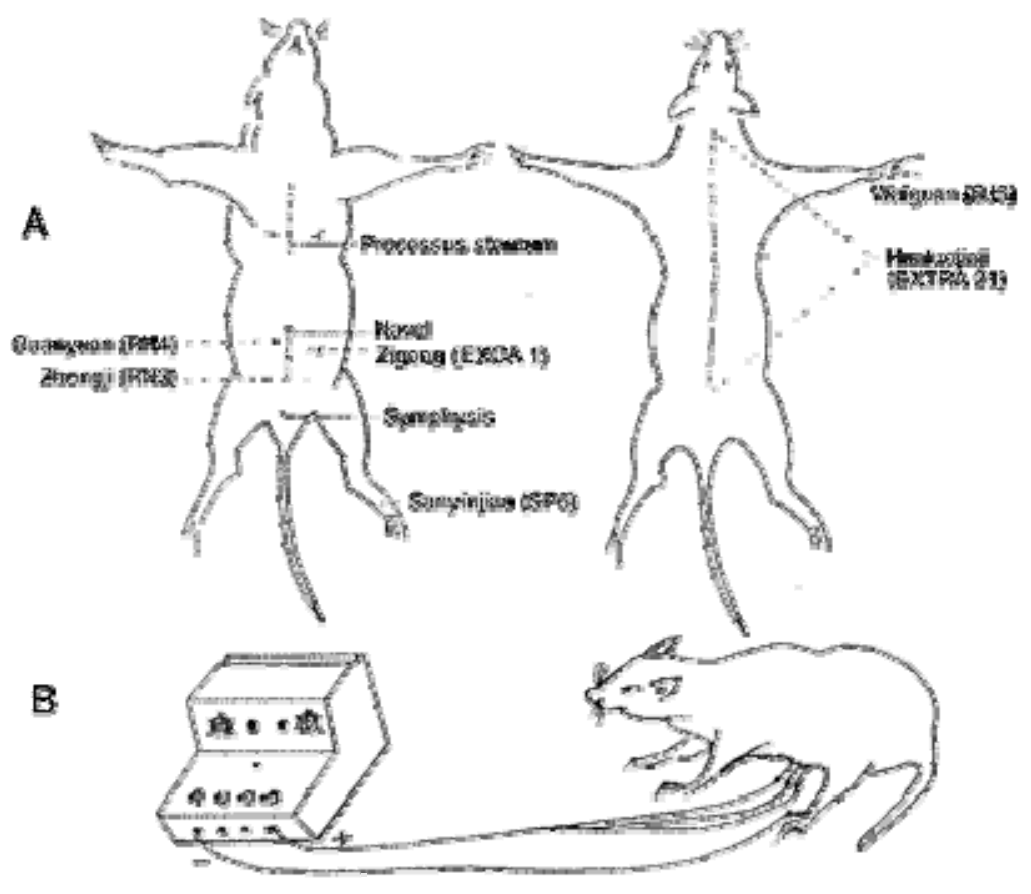

Fig. 1 A: Sketch of ventral view (left) and dorsal view (right) of rat shows the acupoints we used B: Diagram shows the electroacupuncture procedures in conscious rat

Staining techniques: Vaginal smears were fixed by $100 \%$ ethyl alcohol, then stained with $\mathrm{HE}$ method. Adrenal sections were cut in $4 \mu \mathrm{m}$ thickness from paraffin blocks and processed with silver nitrate staining technique[11]. In each case, one hundred cells in zona fascicula were examined randomly under 100 -fold oil immersion lens. Numbers and sizes of AgNOR dots were counted and measured.

C-fos protein immunohistochemistry: The inmunohistochemical analysis of c-fos expression in rat brain was perforrned as previously described[11].

Estrogen receptor (ER) protein immunohistochemistry (ABC method): Under sodium pentobarbital anesthesia (50 mg/kg, ip), the animals were perfused via left cardiac ventricle with $100 \mathrm{ml}$ of phosphate-buffered saline (PBS), followed by $300 \mathrm{ml}$ ice-cold fixative containing $4 \%$ paraformaldehyde in $0.1 \mathrm{M}$ phosphate buffer $(\mathrm{pH} 7.4)$. Afterwards, brain was removed with the same fixative for one day and immersed in 0 . IM phosphate buffer containing $30 \%$ sucrose for another day. The hypothalamus blocks were frozen with dry ice and cut into $35 \mu \mathrm{M}$ thick section by cryostat. The brain sections were washed with $0.01 \mathrm{M}$ PBS for $15 \mathrm{~min} \times 3$ and incubated in $0.01 \mathrm{M}$ PBS containing $0.5 \%$ Triton 100 and $3 \%$ normal goat serum (NGS) at $37^{\circ} \mathrm{C}$-for one hour. Afterwards, the sections incubated in 1:1,000 ER monoclonal antibody $\left(\mathrm{H}_{222}\right.$, Abott Co.) at $37^{\circ} \mathrm{C}$ for one hour, then at $4^{\circ} \mathrm{C}$ for two days. The sections, washed in PBS three times, were processed by $A B C$ kit (from Vecot Labs) induding sequential incubation at $20^{\circ} \mathrm{C}$ in the following solutions with washes between them. (1). second antibody (dilution 1:100), 30min. (2). A+B reagents (dilutionl:100), 60min. (3). $0.05 \%$ diaminobenzidine/ $0.02 \%$ hydrogen peroxide in $0.1 \mathrm{M}$ Tris- $\mathrm{HCl}$ buffer $(\mathrm{pH} 7.2) 10 \mathrm{~min}$. The sections were washed in tap water, mounted and examined under light microscope. The certain areas of typical immunoreactive positive neurons were measured by computer image analysis system (Vecta PC). 
ER mRNA hybridization: The total mRNA of brain was eluted by the modified phenol method [12]. ER cDNA probe (244bp) was labeled by the DIG-labeling kit (from Bohringman Co., Germany). The dot blot hybridization was processed as the method described by Sambrook J and his colleagues [13]. The dot blot images were analyzed with gray density by computer imaging analysis software (TJTY-300, from Tong -Ji university, Shanghai, China).

Statistics: All data in this paper were treated with analysis of variation (ANOVA), least significant difference (ISD) or student T-test.

\section{RESULTS}

\section{Effect of EA on ovulatary induction and curing sterility in woman}

After EA the blood B-EP level of the patients resulting in ovulation either declined or maintain at the levels within the range of the normal levels and the B-EP levels of those failing to show ovulation were significantly higher than the normal's' (table 1). On the other hand, the blood LH and FSH levels of the patients with ovulation after EA treatment tended to be the normal [14].

Table 1. Change of blood ß-EP level before and after EA (pg/ml)

\begin{tabular}{cccc}
\hline Group of cases & N & Before EA & After EA \\
& & & \\
\hline Ovulation & 6 & $65.59 \pm 24.15$ & $\star 38.86 \pm 10.11$ \\
No ovulation & 7 & $65.59 \pm 24.15$ & $80.09 \pm 22.16$ \\
Control & 5 & $38.84 \pm 10.13$ & $41.52 \pm 6.40$ \\
& & & \\
\hline
\end{tabular}

The values in this table are mean \pm SE, ${ }^{*} \mathrm{P}<0.05$

Effect of EA on dysfunction of HPOA in ovariectomized rats

For a further study of the mechanism of EA effect on HPOA a series of experiments in the animal models was performed.

\section{(1). EA induces maturation and exfoliation of vaginal epithelium cell and enhances blood level of} E2.

After ovariectomy two weeks late, the exfoliated epithelium cell disappeared from the vaginal smears of the rats, but it reappeared in the smears following EA treatment. The blood level of E2 in OVX was increased significantly (table 2). No obvious change was seen in INT after EA treatment and in OVX following EA treatment with the control acupoints.

Table 2. The level of blood E2 following EA treatment (pg/ml)

\begin{tabular}{cccc}
\hline Group & $N$ & Before EA & After EA \\
\hline OVX & 10 & $* 5.47 \pm 0.63$ & $* \star 11.58 \pm 0.98$ \\
INT & 10 & $18.00 \pm 3.26$ & $18.34 \pm 8.77$ \\
& & \\
\hline${ }^{*} \mathrm{P}<0.05$ compared with INT, ${ }^{* * P} \mathrm{P}<0.01$ compared with before EA
\end{tabular}

(2). EA promotes enlargement of adrenals and enhances activity of adrenal AgNORs as well as blood level of corticosterone

We found the adrenals of OVX+EA were enlarged and the weight of the adrenals was raised 
significantly. Using histochemical method, the AgNORs of the cells in inner adrenal cortex were examined. The result shows that the activity of AgNORs of OVX was enhanced (table 3,4), and the level of blood corticosterone in OVX+EA was also increased (table 5). There were no similar effects in INT following EA treatment and in OVX after EA with control acupoints.

Table 3. AgNORs number in OVX and INT

\begin{tabular}{cccccc}
\hline $\begin{array}{c}\text { Group } \\
\mathrm{N}\end{array}$ & INT & INI+EA & OVX & OVX+EA & F value \\
& 4 & 3 & 4 & 7 & \\
\hline Number & 1.55 & 1.19 & 1.25 & 2.53 & \\
of AgNORs & 1.82 & 1.28 & 1.61 & 2.05 & \\
(mean/100 cells) & 1.24 & 1.16 & 1.66 & 1.82 & $9.614^{*}$ \\
& 1.30 & & 1.96 & 2.86 & \\
& & & & 2.86 & \\
& & & & 3.93 & \\
& & & & & \\
\hline
\end{tabular}

${ }^{*} \mathrm{P}<0.01$ tested with ANOVA

Table 4. Weight of adrenal

\begin{tabular}{cccccc}
\hline $\begin{array}{c}\text { Group } \\
\mathrm{N}\end{array}$ & $\begin{array}{c}\text { INT } \\
5\end{array}$ & $\begin{array}{c}\text { INI+EA } \\
3\end{array}$ & $\begin{array}{c}\text { OVX } \\
5\end{array}$ & $\begin{array}{c}\text { OVX+EA } \\
8\end{array}$ & F value \\
& & & & & \\
\hline Weight & 57 & 54 & 45 & 67 & \\
(mg) & 56 & 57 & 68 & 72 & \\
& 57 & 58 & 56 & 66 & $5.825^{\star}$ \\
& 43 & & 50 & 71 & \\
& 57 & & 58 & 57 & \\
& & & & 74 & \\
& & & & 68 &
\end{tabular}

${ }^{*} \mathrm{P}<0.01$ tested with ANOVA

Table 5. The levels of blood corticosterone in OVX and INT (mean \pm SE, ng/ml)

\begin{tabular}{cccc}
\hline Group & $\mathrm{N}$ & Before EA & After EA \\
\hline OVX & 12 & $4.78 \pm 0.42$ & ${ }^{*} 6.06 \pm 0.73$ \\
INT & 12 & $3.64 \pm 0.15$ & $4.76 \pm 1.25$ \\
& & \\
\hline${ }^{*} \mathrm{P}<0.001$ compared with before EA &
\end{tabular}

(3). EA decreases the level of hypothalamic $\mathrm{GnRH}$, pituitary $\mathrm{LH}$ and increases the contents of hypothalamic and pituitary ß-endorphin

After EA treatment the levels of $\mathrm{GnRH}$ released from hypothalamus was rnarkedly decreased however, the $ß$-endorphin (ß-EP) secretion in hypothalamus was raised. The pituitary content of $\mathrm{LH}$ was also fallen, but the $\mathrm{B}-\mathrm{EP}$ of pituitary was increased, as well as peripheral $\mathrm{LH}$ and $\mathrm{B}-\mathrm{EP}$ 
level (Fig.2).
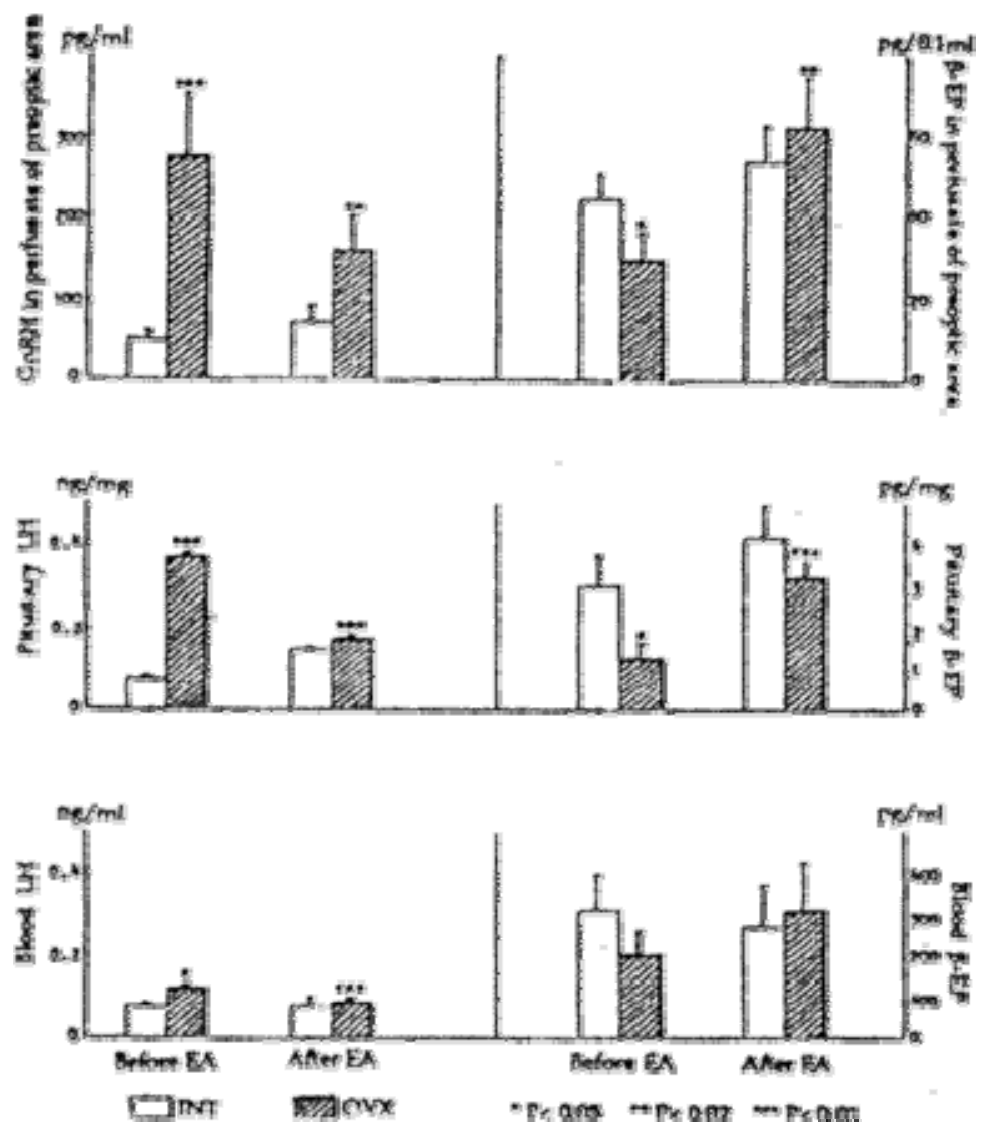

Fig. 2 Change of hypothalarnic GnRH and ß-EP, pituitary LH and B-EP, blood LH and ß-EP before and after EA

Effect of EA on brain c-fos expression in ovariectomized rats

The area occupied by FOS protein labeled neuron was detected in medial preoptic nucleus (MPN), lateral preoptic nucleus (LPN), suprachiasmatic nucleus (SCN), paraventricular nucleus of the hypothalamus (PAVN), medial amygdala nucleus (MAN), periventricular nucleus of the hypothaLsmus (PVN), ventromedial nucleus of the hypothalamus (VNH) and arcuate nucleus (AR) 4 hours after ovariectomy (fig. 3a). The C-fos immunoreactive labeled neurons disappeared two weeks later following ovariectomy. The rats recovering for more than two weeks after ovariectomy, were received EA treatment. Many specific FOS labeled cells were observed in LPN, VNH, SCN and especially in POA, ARN, and PVN, but not any labeled neuron could be found in MAN. No obvious C-fos expression was shown in those nuclei in INT and INT+EA (fig. 3b). 


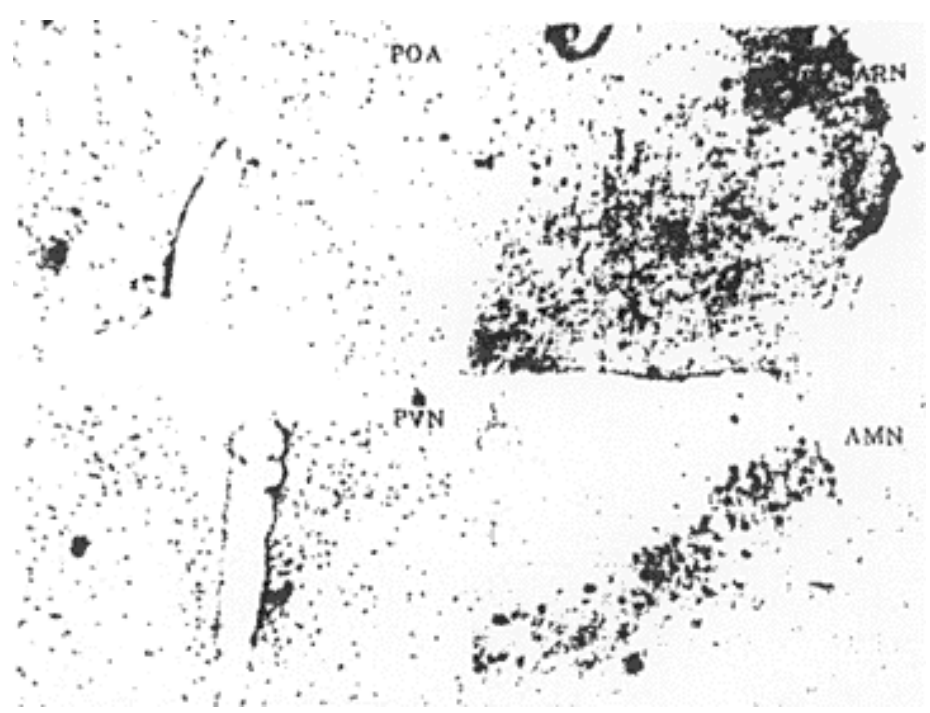

Fig. C-fos immunocytochemistry neurons distribution after ovariectomy $3 a$

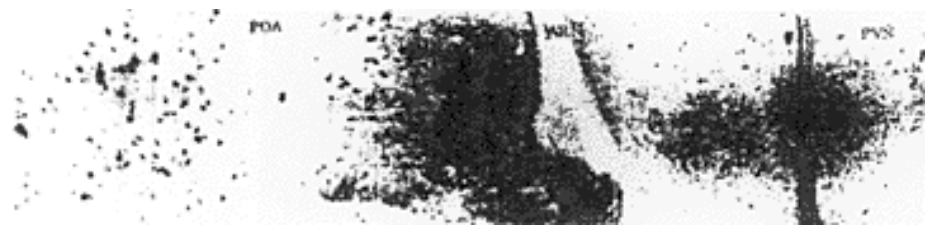

Fig. C-fos expression labeled neurons following electroacupuncture $3 b$

Effect of EA on expression of ER protein and ER mRNA in rat brain Estrogen receptor (ER) immunoreactive neurons were observed widely in rat brain with immunohistochemical technique, especially in MPN, ARN and VNH. The above nuclei were measured by computer image analysis system, and the results show that the mean gray density in OVX+EA was decreased apparently compared with that in OVX. Whereas there were no obvious changes of gray density levels in INT and INT+EA (fig, 4).

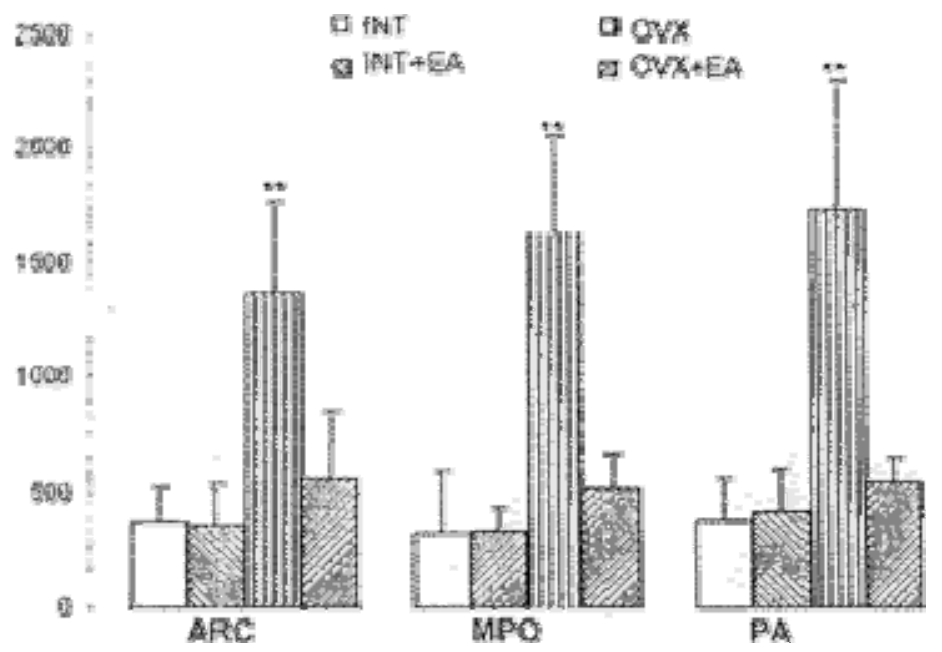


Fig. 4 Effect of EA on expression of ER protein in rat brain (Immunohistochernistry of monoclonal antibody) ${ }^{*} p<0.01$ compared with OVX

The dot blot indicated that ER mRNA expression was increased about 48.11\% in OVX compared with INT. The gray density of OVX was $129.75 \pm 12.13$ and that in OVX+EA was $199.25 \pm 5.75$ attenuated significantly (Fig. 5). The gray density level in INT was $87.60 \pm 5.91$, and the level in INT+EA was $83.60 \pm 4.83$. There was no significant difference between INT and INT+EA
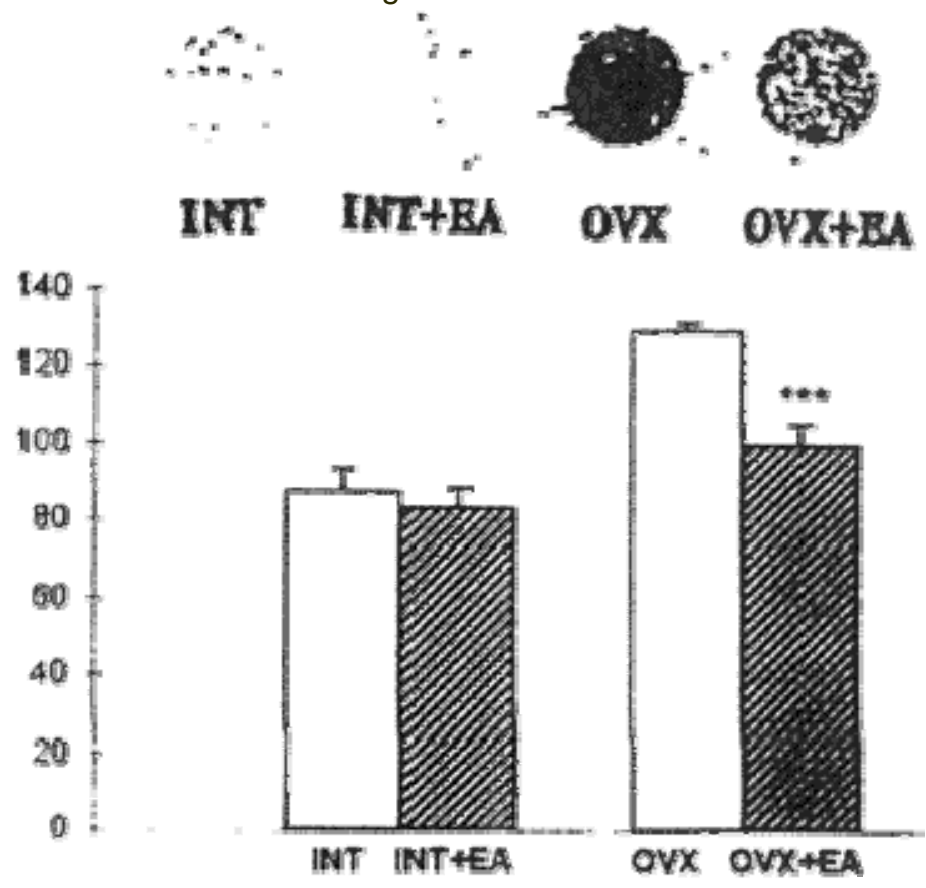

IMT INT +EA OVX OVX $+B A$

Fig. 5 Effect of EA on expression of ER mRNA in rat brain (dot blot) ${ }^{* \star *} p<0.01$ compared with OVX

\section{DISCUSSION}

Since 1985 we have observed that the effect of EA ovulatary induction might relate to the hand skin temperature (HST) and the blood level of B-EP [14]. On the other hand, after EA the blood FSH and LH levels of the patients who successfully ovulated either declined or maintained at normal. In general, provided that body temperature was normal and the environmental temperature was constant round $25^{\circ} \mathrm{C}$, the HST may reflect the state of sympathetic system of a patient. These results suggest that in anovulatary cases the hyperactive sympathetic system can be depressed by EA and the function of HPOA can be regulated by EA through central sympathetic system. Moreover, EA may mediate the abnormal function via the influence on the secretion of the hormones in the different Level of HPOA.

To gain more evidences, we designed some animal experiments to explain the mechanism of EA effects on HPOA at the whole, cellular and molecular levels. We found that EA can induce maturation and exfoliation of vaginal epithelium cell in OVX rat. It is known that maturation and exfoliation of vaginal epithelium cells are a reaction dependent on estrogen level. So we determined the level of blood E2 in OVX and OVX+EA. The result shows the level of blood E2 in OVX was lower than that in normal, but it was increased significantly after OVX accepted EA treatment with the experimental acupoints. This result suggests EA might promote the activity of the compensative mechanism to elevate the subnormal level of E2 induced by ovariectomy in 
rats.

What is this compensative mechanism? To resolve this question, we considered that adrenal is the main organ to secrete sexual hormones except ovarian in females and observed the adrenals of the animals in four groups. The results show that the mean weight of the adrenal in OVX+EA was higher than that in OVX, INT and INT+EA, suggesting the adrenal function might be activated by EA. Subsequently, we detected that the number of AgNORs in zona fasciculata of OVX+EA was significantly increased. Nucleolar organizer regions (NORs) are loops of DNA, which possess ribosomal RNA (rRNA) genes. They are of vital significance in the ultimate synthesis of protein. Thus, the number and configuration of AgNORs (NORs stained by silver staining method) may reflect the activity of cell differentiation and transcription of nucleolar rDNA [15]. In the same time we found the content of blood corticosterone in OVX+EA was raised markedly, but there was no change of blood corticosterone in OVX, INT and INT+EA. This result provided a further evidence that the adrenal cortex cells were initiated in OVX+EA.

The results including the changes of $\mathrm{GnRH}$ releasing from hypothalamus and of the pituitary and blood LH contents suggest that the effects of acupuncture in the regulation of HPOA may be exerted via to promote the function of hypothalamic pituitary-adrenal axis (HPAA), increasing the synthesis and secretion of adrenal steroid horrnones, the androgen of which then be transformed into estrogen in other tissues and thereby reset the negative feedback of estrogen to HPOA. Moreover, EA may accelerate the release of brain and pituitary ß-EP to inhibit the overnormal secretion of $\mathrm{GnRH}$ and LH that may be normalized.

Recently immunohistochemical analysis of the expression of oncogene c-fos $A B I$ was induced by variety of stimuli $[16,17]$. This represents a new method for mapping neuronal activity at the cellular level [18] and thus functionally and systematically tracing neuronal pathway in the nervous system (C NS) [19]. We used this method to examine the distribution of FOS labeled neuron in CNS for recovery of more evidences that EA may alter the neuroendocrine function of HPOA in ovariectomized rats in cellular and gene level. The results show that the specific FOS labeled neurons were observed especially in POA, ARN and PVN in OVX following EA treatment. In above nuclei there were a high concentration of GnRH and B-EP neuron [20]. These results suggest this fact that the expression of FOS labeled neurons reappeared in above mentioned areas following EA treatment in ovariectomized rats may be related to the changes of GnRH and ß-EP from rat hypothalamus after EA treatment.

The level of estrogen in the body may regulate the expression of ER, which may by downregulated following increase of estrogen level and up-regulated after decrease of estrogen [22]. Our finding that after decline of blood E2 induced by ovariectomy the expression of ER was increased and the expression of ER was inhibited by EA inducing the elevation of blood E2 are in accordance with these reported results. ER existing in the brain, especially in POA, ARN and VHN may mediate the function of neuroendocrine system [22, 23]. Thus, our observations suggest that the influence of EA on the change of ER expression in brain may be one of further mechanisms of EA normalizing the dysfunction of HPOA.

INT rats as experimental control we adopted were all of in the stage of preestrus and estrus because the animal sexual hormes and brain ER expressions were changed with the sexual cycle [24]. All INT rats were selected to fix in the two stages there may be a relative constant comparability.

Our results show no same effects were seen after EA treatment in INT and following EA with control acupoints in OVX, suggesting that EA may possess a relative specificity on acupoint and the effect of EA may be a kind of normalization.

\section{CONCLUSION}


Our observations reveal that acupuncture may regulate the abnormal function of HPOA in many ways, which means that acupuncture may activate C-fos expression of brain, then a long term changes at molecular level would start, following the regulation of gene expression in FOS relative gene, such as ER mRNA and GnRH mRNA involved. On the other hand, EA may promote the activity of the body compensative mechanisms, then the levels of hormones, such as $\mathrm{GnRH}, \mathrm{LH}$, estrogen and so on would be normalized. The effect of acupuncture on regulating the function of HPOA may possess a relative specificity of acupoint. Moreover, our clinical and animal experimental results suggest that it is necessary for obtaining a satisfactory effect that proper stimulation should be about thirty minutes Q.D. for three days. This suggestion provides a successful consideration for clinical practice in curing the woman patients with dysfunction of sexual endocrine, such as primary ovarian dysfunction, climacteric syndrom, after-ovariectomy and polycystic ovarian disease etc.

\section{ACKNOWLEDGMENT}

The work was supported by National Natural Foundation of China (3880910 and 392708340) and a grant from the State Key Laboratory of Medical Neurobiology of China (92003).

\section{REFERENCES}

1. Chen, BY et al, Correlation of pain threshold and level of beta-endorphin like immunoreactive substance in human CSF during electroacupuncture analgesia. Acta Physiologica Sinica (in Chinese), 34(4), 385-391, 1984

2. Riahard, ss et al, Electroacupuncture analgesia could be mediated by least two painreleasing endorphins and one endorphin. Life Science, 25, 1957-1968, 1980

3. Yu, J et al, Relationship of hand temperature and blood ß-endorphin immunoreactive substance with electroacupuncture induction of ovulation. Acupuncture Research (in Chinese), 11(2), 86-90, 1986

4. Liu, WC et al, The influence of acupuncture on serum hormones of dysfunction uterine bleeding. Chinese Acupuncture and Moxibustion (in Chinese), 11(5), 37-38, 1991

5. Zhou, $\mathrm{CH}$ et al, Experimental study of the mechanism of acupuncture inducing ovulation. $\underline{\mathrm{J}}$ Combining Chinese and Westen Medicine (in Chinese), 6(12), 764, 1986

6. Shatina, GV et al, Corretive effect of reflextherapy on the hypophyseal-ovarian and sympathetic-adrenal system after ovariectomy. Akush Ginekol MOsk (in Russian), 10, 5861,1991

7. Zheng, W et al, Electroacupuncture-induced acceleration of proopiomelanocortin mRNA in the pituitary and proenkephalin mRNA in the adrenal in rat. Chinese J Physiological Sciences, 3, 106-108, 1986

8. YU, YH et al, Time course of alteration of proopiomelanocorting mRNA level in rat hypothlamic arcuate nucleus following electroacupuncture. Acta Academiae Medicinae Shanghai, 21(Suppl.), 59-62, 1994

9. Nett, TM et al, Aradioimmunoassay for gonadotropin-releasing hoemone $(\mathrm{GnRH})$ in serum. $\underline{\mathrm{J}}$ Clinical Endocrine and Metabolism, 36, 880-883, 1973

10. Howell, WM and Black, DA, Controlled silver-staining organizer reginos with protective coppoidal developer: A 1-step method. Experiment, 36, 1014-1016, 1980

11. $\mathrm{Wu}, \mathrm{ZT}$ et al, The change of $\mathrm{c}$-fos expression in ovariectomized rats following electroacupuncture treatment-An immunohistochemistry study. Acupuncture \& ElectroTherapeutics Research The International Journal, 18, 117-124, 1993

12. Stallcup, MR and Washington, LD, Region-specific initiation of mouse mammary tumor virus RNA synthesis by endogenous RNA polymerase II in preparations of cell nuclei. $\underline{\text { J Biologic }}$ Chemisty 258, 2802-2904, 1083

13. Sambrook, J et al, Molecular Cloning-A Laboratory Manual. 343-355, 2nd edition, Cold Spring 
Harbor Laboratory Press, 1989

14 Chen, BY et al, Relationship between blood radioimmunoreactive beta endorphin and hand skin temperature during the electro-acupuncture induction of ovulation. Acupuncture \& ElctroTherapeutics Research The International Journal 16(1), 1-5, 1991

15. Crocker, C and Paramyit, NAR, Nucleolar organizer regions in lymphomas. J Pathology, 155, 111-118, 1987

16. Omura, $Y$ et al, Simple non-invasive mapping of pain pathway in living humans, and the effect of acute non-invasively induced pain on substance $P$, oncogen C-FOS Ab1, oncogen C-fos Ab2, dopamine and acetycholine. Acupuncture \& Electro-Therapeutics Research The International Journal, 17(4), 291-300, 1992

17. Morgan, $\mathrm{TI}$ et al, Mapping patterns of C-fos expression in the central nervous system after seizure. Science, 237, 192-199, 1984

18. Sagar, S et al, Expression of C-fos protein in brain: Metabolic rnapping at cellular level. Science, 240, 1326-1331, 1988

19. Dragunow, $M$ and Full, $R$, The use of $C$-fos as a metabolic marker in neuronal pathway tracing. J Neuroscience Method, 29, 251-265, 1989

20. Micheal, KS and Harold, GS, Inhibition of hypothalamic-gonadotropin-releasing hormone release of endogenous opioid peptides in the female rabbit. Neuroendocrinology, 46, 14-21, 1987

21. Lauber, $\mathrm{AH}$, et al, Estrogen receptor mRNA expression in rat hypothlamus as a function of genetic sex and estrogen dose. Endocrinology, 129, 3180-3186, 1990

22. Medhabanada, S et al, immunohistochemical localization of estrogen receptor in rat brain, pituitary and uterus with monoclonal antibody. Steroid Biochemistry, 24, 497-503, 1986

23. Simerly, RB, Distribution of androgen and estrogen receptor mRNA containing cell in rat brain an in situ hybridization study. J Comparative Neurology, 294, 76-95, 1990

24. Shughrue, PJ et al, Estrogen receptor mRNA in female rat brain during estrous cycle, a comparision with ovariectomized female and intact male rat. Endocrinoloy, 131, 3180-3186, 1992 